manche. Ce projel grandiose porle l'empreinte des conceptions audacieuses auxquelles notre collègue, $M$. Wilhem, nous avait préparés par ses beaux projets de régularisation du Verdon et de la Durance. Il est extrêmement séduisant et nous ne doutons pas que l'avenir montre qu'il est aisément réalisable et susceptible d'apporter au système hydro-électrique de notre région non seulement les moyens de régularisation désirés, mais encore des possibilités de développement considérable dans l'avenir.

\section{Programme a Établir}

Pour aboutir rapidement, j'estime qu'il est nécessaire d'établir nn programme sur lequel tous les intéressés soient d'accord.

Une des premières données de ce programme doit être la fixation approximative de la quantité d'énergie complémentaire dont nous avons un besoin immédiat. On cherchera en second lieu à établir celle nécessaire pour franchir une seconde étape dans l'aménagement des forces des vallées du Drac et de la Romanche et enfin, on pourrait indiquer le chiffre maximum d'énergie complémentaire que nécessiterait l'aménagement hydraulique intégral de ces vallées.

Nous avons tenu à indiquer l'ordre de grandeur des besoins immédiats de notre région des Alpes.

A cet effet, nous avons pris l'ensemble des usines existantes alimentant les réseaux de distribution du département de l'Isère en laissant de côté pour le moment ceux qui ont déjà leurs moyens propres de régularisation, tels que les réseaux des Sociélés de Force et Lumière, de l'Eau-d'Olle et de Loire et Centre et aussi ceux du bas Grésivaudan qui peuvent être facilement régularisés soil par l'aménagement des Sept Laux, soit par un aménagement partiel des lacs de Belledonne.

Cel ensemble comprend neuf usines, savoir : toutes celles du réseau de Fure et Morge et de la Société d'éclairage de la ville de Grenoble, auxquelles nous avons adjoint deux usines de la vallée de la Romanche, celle de Séchilienne et celle des Vernes et une usine sur le Drac, celle de la Société Drac Romanche, qui sont susceptibles de donner un appoint immédiat de force aux centres industriels qui nous occupent.

Cet ensemble qui représente un total de environ 50.000 HP aménagés, présente d'un bout de l'année à l'autre des variations qui limitent pour le moment son coefficient d'utilisation et font perdre une quantité considérable d'énergie pendant les périodes de hautes eaux.

Néanmoins, le graphique de la figure $n^{\circ} 1$, comparéà celui d'une usine isolée (fig. $n^{0} 2$ ), montre que la coordination de toutes ces usines produit déjà une régularisation appréciable.

Le graphique dont il s'agit et qui donne les puissances mensuelles pendant deux années types, l'une très humide él l'autre très sèche, fait ressortir qu'il existe encore malgré cette coordination des différences importantes entre les puissances mensuelles extrêmes. Cet écart va de $37.000 \mathrm{Kw}$, puissance instantanée totale obtenue pendant le mois de mai de l'année humide, à 13.000 $\mathrm{Kw}$, puissance instantanée obtenue pendant le mois de janvier de l'année très sèche. Cet écart serait encore plus grand si l'on prenait les puissances journalières.

Si l'on cherche à régulariser cette puissance totale en vue d'obtenir une puissance de $30.000 \mathrm{Kw}$ disponible toute l'année, on voit qu'il serait nécessarre d'avoir une usine d'énergie complémentaire fonclionnant en moyenne pendant huit mois par an et capable de donner une pointe instantanée d'au moins 17.000 $\mathrm{Kw}$, probablement 20.000 . Cette pointe pourrait cependant être quelque peu diminuée par le développement des accumulations journalières dans les usines exislantes.

Une première approximation rapidement faite permel de voir que le déficit d'énergie à combler serail d'environ $69.000 .000 \mathrm{de}$ Kwh en année sèche, et de 27.000 .000 de Kwh dans une année humide, mais il faut observer qu'en raison de son prix élevé, cette énergie complémentaire ne serait probablement demandée que par les industries qui onl besoin d'une alimentation régulière, lesquelles sont en général les industries de jour, on peut donc admettre que les besoins immédiats probables du réseau dont il s'agit varieraient de :

23 millions de Kwh pour les années sèches, à 9 millions de Kwh pour les années humides.

L'usine devrait être aménagée pour une puissance maximum qui serait probablement comprise entre 15.000 et $20.000 \mathrm{Kw}$.

\section{ConcLusion}

Ces chiffres, que nous donnons à titre d'indication, devraient être établis plus exactement à la suile d'une élude très précise, et nous terminerons en formant les vœux que ce Congrès provoque la formation d'un ocmité régional comprenant des représentants de l'industrie, des techniciens de la houille blanche, des géographes, des géologues, et des délégués des Sociétés touristiques. Cette réunion d'experts aurait pour mission d'établir sur des données précises les besoins immédiats el futurs de notre région en énergie complémentaire. Elle aurait égalcmenl à faire un premier examen des différents projels que nous avons énumérés plus haut et de tous ceux qui pourraient être présentés dans le même but, et de définir les conditions auxquelles chacun d'eux devrait salisfaire pour rentrer dans le cadre du programme établi et surtout pour ne pas porter d'alleintes graves aux autres intérêts régionaux.

Nous pensons qu'une enquête préalable, faite dans de telles conditions, serait indispensable pour établir un plan rationnel d'aménagement qui salisfasse aux besoins de notre région sans soulever dans la suite d'objections qui seraient de nature à retarder sa réalisation. Une telle méthode aurait en outre l'avantage de facilter aux intéressés la réalisalion des moyens financiers indispensables avec toutes les garanties nécessaires.

\title{
État actuel de l'industrie des Câbles électriques à très haute tension.
}

Par G. DECOMBE, Ingénieur, Fondé de Pouvours de la Compagnie générule des Cables ale Lyon.

L'état actuel de l'industrie des câbles électriques à très haute lension a été parfaitement défini au cours de la Conférence Internationale des grands réseaux électriques à haute tension qui s'est lenue à Paris au mois de novembre dernier.

Nous proposons de reprendre ici les conclusions qui se déduisent de la discussion qui a suivi la lecture du rapport présenté sur cetle question.

Les transports d'énergie à haute tension élant généralement réalisés en courant alternatif triphasé nous considérerons plus

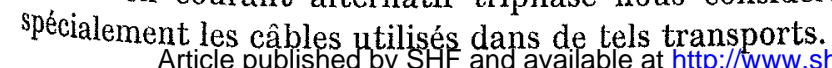

\section{Cables a trots conducteurs}

Les construcleurs paraissent être d'accord pour admettre que le câble à 3 conducteurs présente une sécurité suffisante pour toutes les tensions inférieures à 35.000 volts. De nombreuses installations fonctionnent d'ailleurs d'une façon irréprochable depuis plusieurs années avec des câbles de ce modèle sous dos tensions composées comprises entre 30 et 35.000 volts.

Au delà de 35.000 v. el jusqu'à 50.000 v., l'utilisation des câbles à 3 conducteurs peut être discutée. L'inégalité de la répartition 
des potentiels à la surface de l'ssolant qui recouvre chacun des conducteurs provoque un déplacement continuel des charges statiques sur ces surfaces et donne naissance à des effluves qui détruisent peu à peu le diélectrique lorsque la tension entre conducteurs devient trop grande.

Divers procédés ont été proposés pour supprimer ces effluves en créant artificiellement des surfaces équipotentielles dans les parties de l'isolant où ils prennent naissance.

Cela conduit néanmoins à employer de fortes épaisseurs de papier qui sont difficiles à imprégner. La pose d'aussi gros câbles el la réalisation des boîtes de jonction présentent également d'assez grandes difficultés.

Dans l'état actuel de l'industrie des câbles, nous croyons donc pouvoir affirmer que la tension composée de $60.000 \mathrm{v}$. ne peut être transmise économiquement et en toute sécurité qu'au moyen de câbles à conducteur simple.

\section{CABLES A CONDUCTEUR SIMPLE}

Les câbles à 1 conducteur sous plomb sans armature magnétique se prêtent très bien au transport de grandes quantités d'énergie, sous des tensions élevées. Ils permettent d'utiliser de grosses sections de cuivre tout en conservant au diamètre extérieur du câble el à son poids des valeurs acceptables.

Les surfacés de niveau équipotentiel sont ici des cylindres concentriques; les lignes de force sont dirigées suivant le rayon, en sorte que les couches de papier successives ne travaillent que dans le sens de leur épaisseur. Il en résulle que la tension sous laquelle on peut faire travailler des câbles de ce modèle dépend uniquement de la valeur de la contrainte que l'on peut appliquer au diélectrique.

La contrainte de service généralement admise sur le continent européen pour des câbles de bonne fabrication est comprise entre 4000 el 5000 volts par $\mathrm{m} / \mathrm{m}$, au voisinage du conducteur.

Ces chiffres représentent la valeur minimum de la contrainte que l'on peut admeltre, car malgré tous les soins que l'on apporte à la fabrication du diélectrique, il est à peu près impossible de réaliser un câble ayant sur toute sa longueur un diélectrique parfaitement homogène et ce sont les régions où l'imprégnation est la moins bien faite qui fixent le chiffre que l'on doit adopter.

On peut espèrer que les efforts faits par les constructeurs pour améliorer l'imprégnation de façon à réduire le nombre et l'importance des points faibles, permettront d'adopter une valeur plus élevée pour la contrainte du diélectrique.

En admettant une contrainte inférieure à $5.000 \mathrm{v}$., on a déjà réalisé des canalisations qui fonctionnent sous la tension composée de $60.000 \mathrm{v}$ avec point neutre à la terre.

\section{Utilisation des Cables a Conducteur simple}

Les câbles à un conducteur utilisés pour le transport de l'éner gie sous forme de courant alternatif ne peuvent avoir aucune armature protectrice en sorle que leur fragilité est plus grande que celle des câbles à conducteurs multiples.

Il est donc nécessaire de prendre beaucoup de soins pour ne pas les endommager au moment de leur pose. Il est aussi indispensable de les protéger mécaniquement.

On réalise généralement cette protection en plaçan l les câbles dans des caniveaux en ciment, munis de couvercles.

La position des câbles à l'intérieur des caniveaux n'est pas indifférente, car de cette position dépend le coefficient de selfinduction apparente de chaque conducteur.

A ce point de vue il importe de placer les câbles aussi près que possible l'un de l'autre et de les disposer au sommet d'un triangle équilatéral. Les trois coefficients de self-induction sont ainsi égaux et aussi faibles que possible.

\section{Courant de Circulation}

Malgré cette précaution, la gaîne de plomb qui entoure chacun des conducteurs est le siège d'une force électromotrice qui est fonction de l'intensité qui parcourt le conducteur. Cette force électromotrice produit dans la gaine de plomb un courant de circulation, car le plomb ne peut pas être considéré comme isolé de la terre. Pour éviter les dégâts que pourrait provoquer ce courant aux endroils où le contact avec le sol serait mauvais, la disposition la meilleure paraît être de relier directement le plomb à des plaques de terre de distance en distance et de relier également les plombs des câbles entre eux à chacune des boîtes de jonction. Ainsi les courants induits se trouvent canalisés et ne peuvent produire aucune détérioration dans les plombs des câbles.

La présence de ces courants induits provoque une perte proportionnelle au courant principal ; pour des câbles à $60.000 \mathrm{v}$. 50 périodes, de construction normale, elle est généralement comprise entre $4 \%$ et $8 \%$ de la perte dans le cuivre.

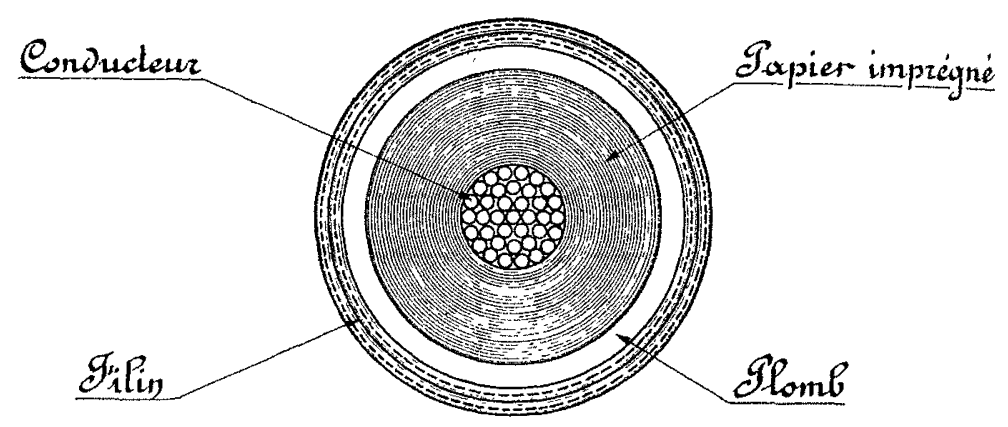

Cable pour transport de force à 60.090 volts.

Pertes diélectriques

On a beaucoup discuté pendant ces dernières années l'importance que peut prendre la valeur des pertes par hystérésis dans le diélectrique. L'étude ce ce phénomène a surtout pour objet de fixer les conditions de travail que l'on peut demander à l'isolant, car il est connu que lorsque l'échauffement produit par hystérésis dans le diélectrique devient trop élevé, le diélectrique esı rapidement détruit. Mais, au point de vue de l'exploitation, l'étude des pertes par hystérésis ne présente un intérêt réel que pour la consommation d'énergie qui en résulte, puisque cette perte est constante.

Nous nous bornerons donc à indiquer que la valeur des pertes dues à l'hystérésis diélectrique ne dépasse pas 900 watts par km de câble simple à 60.000 volts à la fréquence de 50 périodes, et à la température de régime admise de $45^{\circ} \mathrm{C}$.

Cette perte ne produit d'ailleurs qu'un échauffement très faible de l'isolant et ne diminue pas sensiblement la densité de courant que l'on peul admettre dans le conducteur.

\section{Cables pour Courant continu a haute tension}

Les câbles à un conducteur peuvent être également utilisés pour le transport de l'énergie sous forme de courant continu, système série, sous des tensions très élevées. Le diélectrique travaille ici dans des conditions très différentes qu'en courant alternatif, car il n'esl plus soumis au travail hystérétique et c'est seulement le coefficient de rigidité électrostatique qui détermine sa tension de rupture. Les essais qui ont été faits dans ce sens et l'expérience de quelques canalisations qui fonctionnent en courant continu à haute tension depuis plus de quinze années permettent de dire que la contrainte que l'on peut admettre sous lension continue est environ trois fois plus grande que celle que l'on admet sous tension alternative.

Nous pensons qu'il était utile de le signaler en raison du rôle important que le câble souterrain peut remplir lorsque l'on utilise ce mode de transport de l'énergie. 